\title{
Software-type Wave-Particle Interaction Analyzer on board the Arase satellite
}

\author{
Yuto Katoh ${ }^{1 *} \mathbb{D}$, Hirotsugu Kojima², Mitsuru Hikishima ${ }^{3}$, Takeshi Takashima ${ }^{3}$, Kazushi Asamura ${ }^{3}$, \\ Yoshizumi Miyoshi ${ }^{4}$, Yoshiya Kasahara ${ }^{5}$, Satoshi Kasahara ${ }^{6}$, Takefumi Mitani ${ }^{3}$, Nana Higashio ${ }^{7}$, Ayako Matsuoka ${ }^{3}$, \\ Mitsunori Ozaki ${ }^{5}$, Satoshi Yagitani ${ }^{5}$, Shoichiro Yokota ${ }^{8}$, Shoya Matsuda ${ }^{4}$, Masahiro Kitahara $^{1}$ and Iku Shinohara ${ }^{3}$
}

\begin{abstract}
We describe the principles of the Wave-Particle Interaction Analyzer (WPIA) and the implementation of the Softwaretype WPIA (S-WPIA) on the Arase satellite. The WPIA is a new type of instrument for the direct and quantitative measurement of wave-particle interactions. The S-WPIA is installed on the Arase satellite as a software function running on the mission data processor. The S-WPIA on board the Arase satellite uses an electromagnetic field waveform that is measured by the waveform capture receiver of the plasma wave experiment (PWE), and the velocity vectors of electrons detected by the medium-energy particle experiment-electron analyzer (MEP-e), the high-energy electron experiment (HEP), and the extremely high-energy electron experiment (XEP). The prime objective of the S-WPIA is to measure the energy exchange between whistler-mode chorus emissions and energetic electrons in the inner magnetosphere. It is essential for the S-WPIA to synchronize instruments to a relative time accuracy better than the time period of the plasma wave oscillations. Since the typical frequency of chorus emissions in the inner magnetosphere is a few $\mathrm{kHz}$, a relative time accuracy of better than $10 \mu \mathrm{s}$ is required in order to measure the relative phase angle between the wave and velocity vectors. In the Arase satellite, a dedicated system has been developed to realize the time resolution required for inter-instrument communication. Here, both the time index distributed over all instruments through the satellite system and an S-WPIA clock signal are used, that are distributed from the PWE to the MEP-e, HEP, and XEP through a direct line, for the synchronization of instruments within a relative time accuracy of a few $\mu \mathrm{s}$. We also estimate the number of particles required to obtain statistically significant results with the S-WPIA and the expected accumulation time by referring to the specifications of the MEP-e and assuming a count rate for each detector.
\end{abstract}

Keywords: Radiation belts, Magnetosphere, Whistler-mode chorus, Wave-particle interactions

\section{Introduction}

The Arase (ERG) satellite was launched from the Uchinoura Space Center on December 20, 2016 to explore the dynamics of the terrestrial radiation belts. One of the prime objectives for this satellite mission is the investigation of the energization process of relativistic electrons by whistler-mode chorus emissions. Whistlermode chorus emissions are coherent electromagnetic plasma waves observed mainly on the dawn side of the

\footnotetext{
*Correspondence: yuto@stpp.gp.tohoku.ac.jp

${ }^{1}$ Department of Geophysics, Graduate School of Science, Tohoku

University, 6-3 Aramaki-aza-aoba, Aoba, Sendai, Miyagi 980-8578, Japan

Full list of author information is available at the end of the article
}

inner magnetosphere (e.g., Summers et al. 1998). Previous studies showed that chorus emissions play crucial roles in the reformation of the outer radiation belt during the recovery phase of geomagnetic storms (e.g., Miyoshi et al. 2003). Recent theoretical and simulation studies have revealed that chorus emissions emerge from a band of whistler-mode waves in regions close to the magnetic equator through nonlinear wave-particle interactions (e.g., Katoh and Omura 2007, 2011, 2013, 2016; Omura et al. 2008, 2009). Chorus emissions propagate away from the equator, and their propagation characteristics vary depending on the plasma environment in the inner magnetosphere (e.g., Katoh 2014). In the generation process of chorus emissions, an electromagnetic electron hole is 
formed in a specific range of the velocity phase space due to the nonlinear Lorentz force acting on resonant electrons. Simulation studies have revealed that most resonant electrons lose their kinetic energy, contributing to the generation of chorus emissions, and that a fraction of the resonant electrons is trapped inside the hole and is effectively energized through a special form of nonlinear wave trapping called relativistic turning acceleration (Omura et al. 2007) and ultra-relativistic acceleration (Summers and Omura 2007).

Wave-particle interactions in the magnetosphere occur over the timescale of the characteristics of plasma waves and particles. During the interaction between coherent whistler-mode waves and energetic electrons, relaxation of the velocity distribution function of resonant electrons occurs within hundreds or thousands of electron gyro-periods (e.g., Katoh and Omura 2004), corresponding to tens of $\mathrm{ms}$ for typical parameters of the Earth's inner magnetosphere. Since the time resolution of conventional plasma instruments on board a spacecraft is usually a few tens of ms or less, it is difficult to measure the relaxation of the velocity distribution or the energy exchange between wave and particles.

To overcome the difficulty in the direct measurement of wave-particle interactions, previous studies have used the observed wave phase as a reference to count the number of particles in order to obtain the distribution as a function of the relative phase angle between waves and particles (Ergun et al. 1991, 1998; Gough et al. 1995; Buckley et al. 2000). In sounding rocket experiments, their attempts successfully identified wave-particle correlations between Langmuir waves and electrons, with a statistical significance (Kletzing et al. 2017). Fukuhara et al. (2009) proposed a new type of instrument for the direct and quantitative measurement of the energy exchange between waves and particles, which is referred to as the WaveParticle Interaction Analyzer (WPIA). The WPIA uses the three components of observed waveforms and particle velocity vectors to quantify the energy flow by measuring the inner product of the observed instantaneous wave and velocity vectors, corresponding to Joule heating of particles by plasma waves (Katoh et al. 2013). The feasibility of the WPIA for the Arase satellite has been studied using pseudo-observations based on simulations with self-consistent plasma particle codes, which reproduce the process of chorus generation (Katoh et al. 2013; Hikishima et al. 2014). Kitahara and Katoh (2016) suggested that the WPIA is also capable of measuring the pitch angle scatter of particles by plasma waves directly and quantitatively. Recently, Shoji et al. (2017) showed that the WPIA can directly measure the formation of an ion hole through interactions of electromagnetic ion cyclotron waves and energetic ions in the inner magnetosphere.
In this paper, the implementation of the Software-type Wave-Particle Interaction Analyzer (S-WPIA) on the Arase satellite is described. Since the Arase satellite is the first application of the WPIA in space, we installed the Software-type WPIA because of its flexibility in choosing processing algorithms and optimization. The principles and significance of the WPIA are discussed in "Principles of the WPIA and its significance" section. Details of the S-WPIA implementation in the Arase satellite are described in "S-WPIA implemented on the Arase satellite" section, and a summary is present in "Summary" section.

\section{Principles of the WPIA and its significance}

The WPIA proposed by Fukuhara et al. (2009) uses the three components of observed waveforms and particle velocity vectors. The WPIA quantifies the energy flow by measuring the inner product of the observed instantaneous wave electric field and velocity vectors, $\boldsymbol{E}$ and $\boldsymbol{v}$, which is the time variation of the kinetic energy of a charged particle and is given by

$$
W=\frac{\mathrm{d} K}{\mathrm{~d} t}=m_{0} \boldsymbol{v} \cdot \frac{\mathrm{d}(\gamma \boldsymbol{v})}{\mathrm{d} t}=q \boldsymbol{E} \cdot \boldsymbol{v},
$$

where $K=m_{0} c^{2}(\gamma-1)$ is the kinetic energy of a charged particle including relativistic effects, $m_{0}$ and $q$ are the rest mass and charge of a particle, respectively, $c$ is the speed of light, and $\gamma$ is the Lorentz factor. According to Katoh et al. (2013), the net variation of the kinetic energy of charged particles, $\Delta W(\boldsymbol{r}, t)$, during a time interval $\Delta t$ is given by

$\Delta W(\boldsymbol{r}, t)=\int_{t}^{t+\Delta t} \iiint q \boldsymbol{E}\left(\boldsymbol{r}, t^{\prime}\right) \cdot \boldsymbol{v} f\left(\boldsymbol{r}, \boldsymbol{v}, t^{\prime}\right) d \boldsymbol{v} d t^{\prime}$,

where $f$ is the phase space density of charged particles. Since the measurement of $f$ is performed at discrete times, $\Delta W(\boldsymbol{r}, t)$ is discretized as a summation of $W\left(t_{i}\right)=$ $q \boldsymbol{E}\left(t_{i}\right) \cdot \boldsymbol{v}_{i}$ measured over a time interval $\Delta t$, as follows:

$$
\Delta W(\boldsymbol{r}, t) \simeq \sum_{i=1}^{N} q \boldsymbol{E}\left(t_{i}\right) \cdot \boldsymbol{v}_{i}=\sum_{i=1}^{N} W\left(t_{i}\right),
$$

where $t \leq t_{i} \leq t+\Delta t, N$ represents the number of particles detected during the time interval $\Delta t, t_{i}$ is the detection time for the $i$-th particle, $\boldsymbol{E}\left(t_{i}\right)$ is the wave electric field vector at $t_{i}$, and $\boldsymbol{v}_{i}$ is the velocity vector for the $i$-th particle. Since $W\left(t_{i}\right)$ represents the gain or the loss of the kinetic energy of the $i$-th particle, the net amount of the energy exchange in the region of interest is obtained by summing $W$ for all detected particles, where $W_{\text {int }}=\sum_{i=1}^{N} W\left(t_{i}\right)$. Figure 1 shows a schematic diagram of $W$ and $W_{\text {int }}$ as measured by the S-WPIA for interactions between energetic electrons and whistler-mode 
waves propagating purely parallel to the background magnetic field (after Katoh et al. 2013), where $\boldsymbol{E}_{\mathrm{w}}$ and $\boldsymbol{B}_{\mathrm{w}}$ are the wave electric and magnetic field vectors, respectively, and $\boldsymbol{v}_{\perp}$ is the perpendicular component of the velocity vector of a particle. The sign of $W$ is determined by the relative phase angle $(\theta)$ between $\boldsymbol{E}_{\mathrm{w}}$ and $\boldsymbol{v}_{\perp}$ (Fig. 1a, b), and the net energy exchange between particles and waves can be evaluated by summing $W$ for all $N$ particles to obtain $W_{\text {int }}$ (Fig. 1c). By representing the numbers of energetic electrons having positive and negative $W$ by $N_{+}$and $N_{-}$, respectively, it is expected that $N_{+}$ and $N_{-}$would be significantly different from each other in the region of efficient wave-particle interactions. Figure $1 \mathrm{c}$ indicates the case of an efficient wave-particle

(a) $W=\boldsymbol{F}_{E} \cdot \boldsymbol{v}_{\perp}<0$

(b) $W=\boldsymbol{F}_{E} \cdot \boldsymbol{v}_{\perp}>0$
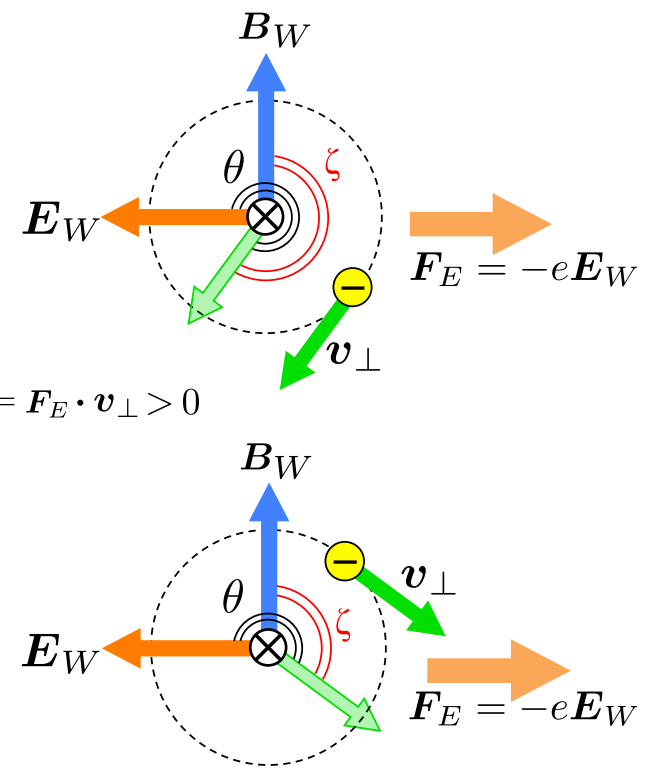

(c)

$\sum_{i=1}^{N} W_{i}=W_{\mathrm{int}}<0 \quad \uparrow n(W)$

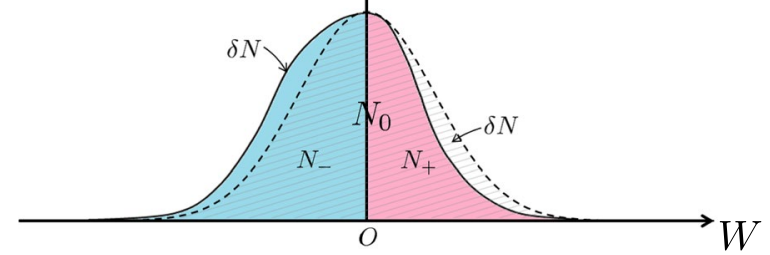

Fig. 1 Schematic diagram of the S-WPIA for measuring interactions between energetic electrons and whistler-mode waves propagating purely parallel to the background magnetic field (after Katoh et al. 2013). Panels $\mathbf{a}$ and $\mathbf{b}$ represent the relation between the perpendicular component of the velocity vector of an electron $\left(\boldsymbol{v}_{\perp}\right)$ and the wave electric $\left(\boldsymbol{E}_{\mathrm{w}}\right)$ and magnetic $\left(\boldsymbol{B}_{\mathrm{w}}\right)$ field vectors in the cases of $\mathbf{a} W$ $<0$ and $\mathbf{b} W>0$. c Distribution of energetic electrons as a function of $W$, corresponding to a negative $W_{\text {int }}$ case representing wave generation. The total number of energetic electrons is $N_{0}$, while $N_{+}$and $N_{-}$ are the numbers of energetic electrons having positive and negative $W$, respectively interaction resulting in a wave generation, where $N_{-}$is larger than $N_{+}$, rendering $W_{\text {int }}$ negative. Alternatively, if the difference $(\delta N)$ between $N_{+}$and $N_{-}$is negligible, $W_{\text {int }}$ approaches zero and no net energy exchange would occur. Since a finite number of particles are used in the computation of $\delta N$ and $W_{\text {int }}$, there is a fluctuation over time. The fluctuation originates from the thermal fluctuation of the distribution of energetic electrons as well as the fluctuation of both wave electric field amplitude and relative phase angle $\theta$. We use the standard deviation $\sigma_{\mathrm{W}}$, which is computed by:

$\sigma_{W}=\sqrt{\sum_{i=1}^{N}\left(q \boldsymbol{E}_{W}\left(t_{i}\right) \cdot \boldsymbol{v}_{i}\right)^{2}-\frac{1}{N}\left(\sum_{i=1}^{N} q \boldsymbol{E}_{W}\left(t_{i}\right) \cdot \boldsymbol{v}_{i}\right)^{2}}$,

where the first and second terms on the right-hand side correspond to the width and the center of the $q \boldsymbol{E}_{\mathrm{W}}\left(t_{i}\right) \cdot \boldsymbol{v}_{i}$ distribution, respectively, to evaluate the statistical significance of the obtained $W_{\text {int }}$ compared to the fluctuation. We can identify an efficient energy exchange between waves and particles when a $W_{\text {int }}$ that is sufficiently larger than the $\sigma_{\mathrm{W}}$ is obtained by the S-WPIA. In other words, a sufficient number of particles need to be collected for the computation of $W_{\text {int }}$, so that the obtained $W_{\text {int }}$ exceeds $\sigma_{\mathrm{W}}$ and achieves the required statistical significance, assuming a Gaussian distribution of $1.64 \sigma_{\mathrm{W}}$ for a statistical significance of $90 \%$ and $1.96 \sigma_{\mathrm{W}}$ for a $95 \%$ scenario. For the case in which a sufficiently large number of particles is expected in the S-WPIA, $W_{\text {int }}$ can be evaluated for different kinetic energy $(K)$ and pitch angle $(\alpha)$ ranges to obtain $W_{\text {int }}(K, \alpha)$. By examining the obtained $W_{\text {int }}(K, \alpha)$, we can identify the specific energy and pitch angle ranges that mostly contribute to the energy exchange through wave-particle interactions. In this case, $\sigma_{\mathrm{W}}(K, \alpha)$ should also be computed for the evaluation of the statistical significance of the obtained $W_{\text {int }}(K, \alpha)$.

\section{Specifications of instruments on board the Arase satellite for implementing the S-WPIA}

For the WPIA, it is essential to ascertain that the time resolution of $t_{i}$, indicating the detection time for the $i$-th particle, is shorter than the timescale for the wave-particle interactions. For the S-WPIA on board the Arase satellite, the requirement of the relative time accuracy for each instrument used in the direct measurement of interactions between the chorus and energetic electrons in the inner magnetosphere is studied. The relative phase angle between the electromagnetic field vector for the wave $\left(\boldsymbol{E}_{\mathrm{w}}\right.$ and $\boldsymbol{B}_{\mathrm{w}}$ ) and the velocity vector $\boldsymbol{v}_{\perp}$ for the energetic electrons should be resolved in order to identify the sign of $W$ correctly for each detected electron. Here, $\theta$ represents 
the relative phase angle between $\boldsymbol{E}_{\mathrm{w}}$ and $\boldsymbol{v}_{\perp}$ (Fig. 1a, b), and $\zeta$ denotes the angle between $\boldsymbol{B}_{\mathrm{w}}$ and $\boldsymbol{v}_{\perp}$. In addition, identifying of the presence of an electromagnetic electron hole in the velocity phase space is one of the primary goals of the S-WPIA. While the hole is formed in the specific range of $\zeta$ (e.g., Omura et al. 2008; Katoh et al. 2013), which rotates in time with the wave period, the wave phase variation needs to be resolved on a timescale that is sufficiently shorter than the wave period. In the inner magnetosphere, chorus emissions appear in a frequency range lower than the electron cyclotron frequency: typically, from 0.2 to $0.5 \Omega_{\mathrm{e} 0}$ for the lower band chorus and from 0.5 to $0.8 \Omega_{\mathrm{e} 0}$ for the upper band chorus, where $\Omega_{\mathrm{e} 0}$ is the electron gyrofrequency at the magnetic equator. Assuming $10 \mathrm{kHz}$ as the highest electron cyclotron frequency along the Arase orbit at the equator, the wave period of the chorus is approximately $100 \mu \mathrm{s}$. An accuracy greater than $10 \mu$ s resolves the wave phase on the order of a few tens of degrees. The same accuracy should be utilized for the synchronization between wave and particle instruments in order to identify $\theta$ and $\zeta$ correctly.

The instruments on board the Arase satellite meet the requirements for direct measurements of interactions between chorus and energetic electrons by the S-WPIA. Chorus emissions are often observed on the dawn side of the inner magnetosphere and outside the plasmapause. The typical frequency range of chorus emissions is covered by the waveform capture receiver (WFC) of the plasma wave experiments (PWE) on board the Arase satellite (Kasahara et al. 2018a). Furthermore, since the ratio between the plasma frequency $\left(f_{\mathrm{p}}\right)$ and the electron cyclotron frequency $\left(f_{\mathrm{ce}}\right), f_{\mathrm{p}} / f_{\mathrm{ce}}$, is typically less than 10 , the minimum resonance energy based on the first-order cyclotron resonance condition is estimated to be in the energy range of hundreds of $\mathrm{eV}$ to a few $\mathrm{keV}$ for the upper band chorus and from a few $\mathrm{keV}$ to tens of $\mathrm{keV}$ for the lower band chorus, respectively. The resonance energy changes depending on the pitch angle of the resonant electrons and increases to over $\mathrm{MeV}$ for large pitch angle ranges. These estimations show that the kinetic energy range of resonant electrons, particularly for the lower band chorus, is covered by the medium-energy particle experiments (MEP-e) (Kasahara et al. 2018b), the highenergy electron instruments (HEP) (Mitani et al. submitted to Earth, Planets and Space), and the extremely high-energy electron experiment (XEP) (Higashio et al. submitted to Earth, Planets and Space) on board the Arase satellite.

Estimation of the required integration time for the S-WPIA For the direct measurements of wave-particle interactions by the S-WPIA, a certain number of particles detected in the region of interest need to be collected in order to obtain a statistically significant $W_{\text {int }}$ and/or a non-uniform distribution of particles in the wave phase space caused by the presence of an electromagnetic electron hole. Assuming that the distribution of energetic electrons as a function of $\zeta$ is changed by $10 \%$ from the average due to the presence of an electromagnetic electron hole and that the statistical fluctuation follows a Poisson distribution for which the standard deviation is expressed as $N^{1 / 2} / N$ for a particle count $N$, at least more than 100 particles need to be collected in each bin. If the distribution of particles as a function of relative phase angle $\zeta$ is analyzed at every $30^{\circ}$, i.e., if 12 bins are assumed for $\zeta$ from $0^{\circ}$ to $360^{\circ}$, then the collection of 1200 particles would be required to assess each of the kinetic energy and pitch angle ranges.

By referring to the specifications of the MEP-e (Kasahara et al. 2018b), the number of particles required for the S-WPIA required to obtain a statistically significant $W_{\text {int }}$ is estimated. The MEP-e measures electrons in the energy range of 5-80 keV using 16 sensor channels, where each sensor has an angular resolution of $5^{\circ}$ for both elevation and azimuth angles. By estimating the expected particle counts for the MEP-e, the observation conditions for the Arase satellite are assumed to be as follows: (1) the background magnetic field is perpendicular to the spin axis of the Arase satellite, and (2) the number of energy steps for the MEP-e is 16, swept four times every second. Since the field-of-view (FOV) for each sensor channel of the MEP-e changes with time due to the satellite spin, the FOV and the corresponding pitch angle for each sensor channel, as well as the energy step for the MEP-e measurement during one spin, are computed as shown in Fig. 2. Figure 2a shows the pitch angle measured by four sensor channels illustrated by colored rectangles in the upper panel, where the same color is used for both lines in Fig. 2a and rectangles indicating the FOV of the corresponding sensor channel. The pitch angle measured by each sensor channel changes in time due to the satellite spin. The coverage of the pitch angle is different depending on the direction of the FOV with respect to the background magnetic field. The energy range measured by each sensor channel also varies in time, as shown in Fig. 2b. Since MEP-e sweeps 16 energy step every $0.25 \mathrm{~s}$, the energy and the pitch angle measured by each sensor vary accordingly. By referring the observation sequence indicated by Fig. 2a, b, we compute the expected count rate during one spin period as a function of both energy and pitch angle of electrons. The flux of incoming energetic electrons to the MEP-e is assumed to be uniform in both time and space during one spin period with a count rate of 5000 counts per second (cps) for each sensor 
channel. Figure 2c shows the estimated particle count as a function of the energy steps and pitch angle bins, where the width of each pitch angle bin is assumed to be $5^{\circ}$. The estimation shows that a particle count greater than 2000 can be expected in the wide pitch angle range from $60^{\circ}$ to $120^{\circ}$ in all the kinetic energy range covered by the MEP-e.

The required time interval for the S-WPIA based on the estimation shown in Fig. 2c is evaluated. If the required number of particles is set at 2000 as estimated earlier, the required particle count can be collected by MEP-e within one spin period in the pitch angle range from $60^{\circ}$ to $120^{\circ}$. However, additional restrictions and limitations should be taken into account for the S-WPIA. If the number of particles required in order to increase the statistical significance of the obtained results is set at 12,000 , the accumulation time should be greater than six spin periods in the pitch angle range from $60^{\circ}$ to $120^{\circ}$. In addition to using a large particle count to achieve statistical significance, in order to increase the signal-tonoise ratio for the S-WPIA, the count at the time of the whistler-mode chorus enhancements should be used. We expect that both the net increase of $\mathrm{W}_{\text {int }}$ and modulation of the particle distribution as a function of the relative phase angle $\zeta$ can only be measured in the presence of chorus emissions. Considering that the statistical fluctuation of the particle count is expressed as $N^{1 / 2} / N$, the particle count detected in the absence of chorus emissions only increases the statistical fluctuations without increasing the amount of the modulation due to wave-particle interactions. By selecting the interval of chorus emissions, we expect that the detected count will increase both $N^{1 / 2} / N$ and the amount of the modulation of the distribution, and therefore, we expect the signal-to-noise ratio to increase. Since chorus elements appear in the spectra intermittently with a timescale of less than $1 \mathrm{~s}$, it can be roughly assumed that one-third of the detected particles are accompanied by chorus elements. Taking these assumptions into account, the required accumulation time for the S-WPIA is estimated to be at least 18 spin periods, corresponding to $144 \mathrm{~s}$. The expected duration of the S-WPIA measurements is more than $3 \mathrm{~min}$ in the region of interest, and this expectation is considered in the operation planning for the Arase satellite.

\section{S-WPIA implemented on the Arase satellite}

In order to realize the S-WPIA in the Arase satellite, a dedicated mission network system for the synchronization of wave and particle instruments was developed. In this section, the implementation of the S-WPIA on the Arase satellite is described.
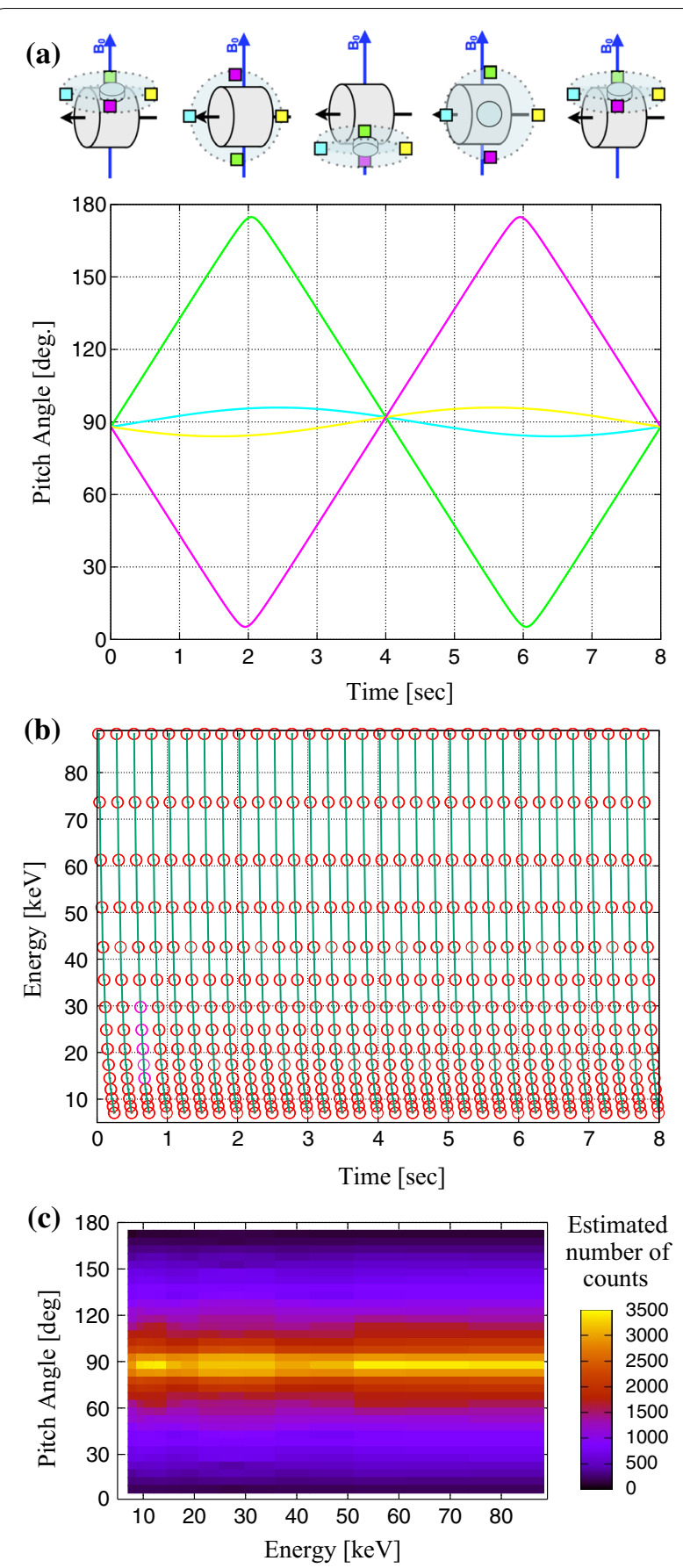

Fig. 2 a Variation of the pitch angle at the center of the field-ofview for sensor channels of the MEP-e during one spin period of $8 \mathrm{~s}$ under the assumed condition. Schematics shown in the upper panel represent the FOV of each sensor channel every $2 \mathrm{~s}$, where the color of rectangles corresponds to those of plotted lines. $\mathbf{b}$ Energy range measured by MEP-e during one spin period, where 16 energy steps are swept every $0.25 \mathrm{~s}$. c Estimated number of counts measured by the MEP-e during one spin period 


\section{Mission network based on the Spacewire}

All the scientific instruments connect to the mission network through dedicated CPU boards, which are digital processing boards designed specifically for the Arase mission. The mission network is a communication system based on the Spacewire standardization (ECSS-E-ST-5012C 2008; ECSS-E-ST-50-51C 2010; ECSS-E-ST-50-52C 2010). The scientific instruments communicate with each other and transfer the observed data to the mission data recorder (MDR) (Takashima et al. submitted to Earth, Planets and Space). The MDR is composed of a CPU with $128 \mathrm{MB}$ of SD-RAM and a 32-GB flash memory. The flash memory in the MDR is dedicated to the storage of the data related to the S-WPIA and the PWE burst mode (Kasahara et al. 2018a). The S-WPIA application software, which runs on the MDR, executes WPIA calculations and manages the data flow on the MDR (Hikishima et al. submitted to Earth, Planets and Space). Figure 3 shows the configuration of the mission network. Note that the components in the relation to the S-WPIA are shown in this figure. While the XEP, HEP, MEP-e, and MGF connect to the mission network through their own set of the CPU boards, the PWE connects through two sets of CPU boards, one of which is dedicated to the data management for the electric field channels, and the other of which is dedicated to the data management for the magnetic field channels.

Each scientific instrument writes the observed data into the MDR in the S-WPIA data format through the mission network. The communication among the scientific instruments is conducted by a relay packet. The data packed in the relay packet are transferred by each instrument according to the routing information, which is decided in advance by commands. Through the relay packet, the S-WPIA activates the generation of the data designated to the S-WPIA for each instrument. Each instrument reports its readiness and generation statuses for the S-WPIA data through the relayed packet. The total bandwidth of the mission network is $12 \mathrm{Mbps}$, and a specific bandwidth is allocated to each instrument based on the bit rate of the data generation by commands.

\section{Accuracy of relative observation time}

As stated in "Principles of the WPIA and its significance" section, the application of the S-WPIA to the chorus emission requires an accuracy of at least $10 \mu \mathrm{s}$ for the relative observation time among the plasma wave receiver and the particle instruments. Since the observation times for each

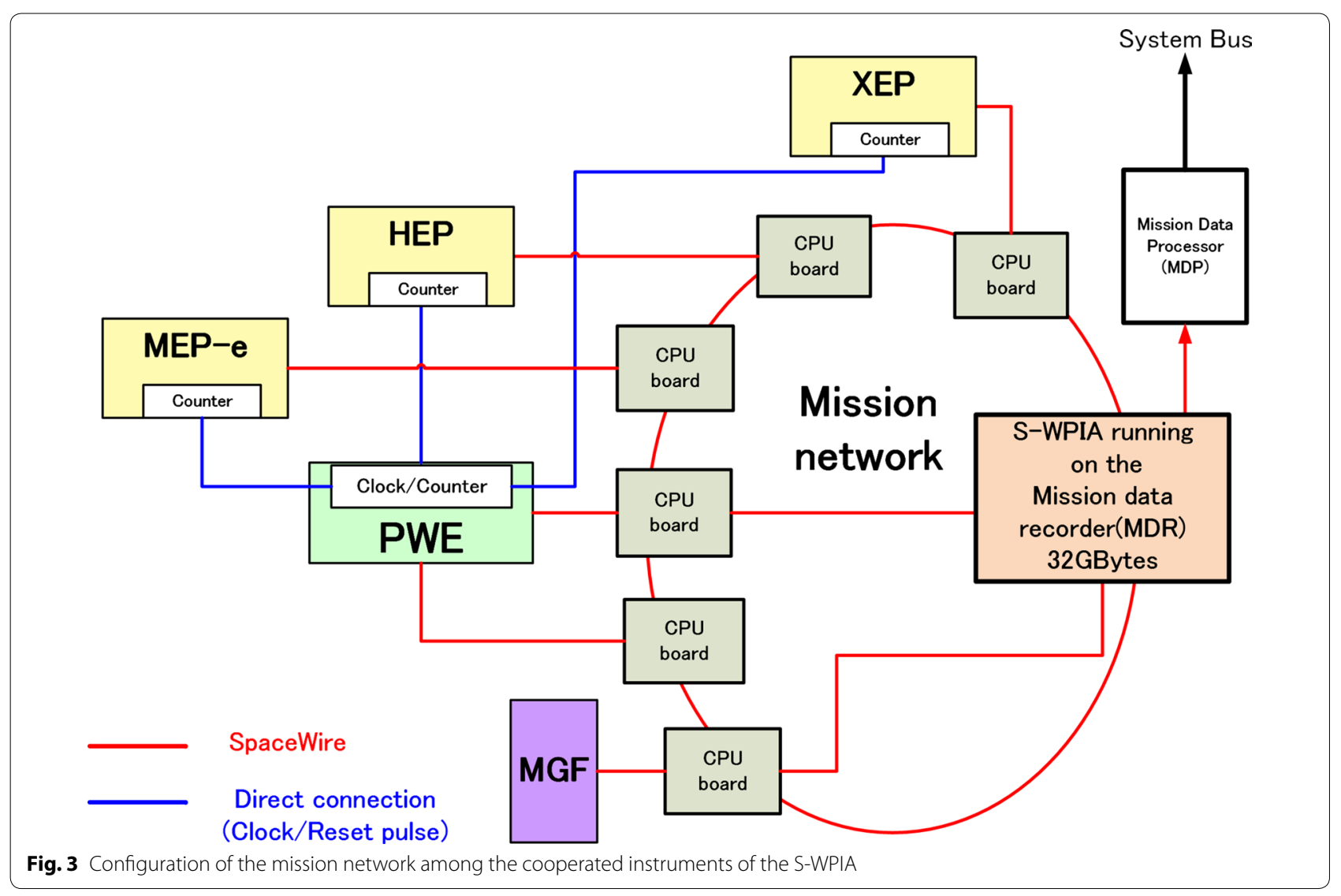


Satellite time (time resolution of $15.6 \mathrm{~ms}$ ) distributed via space wire network
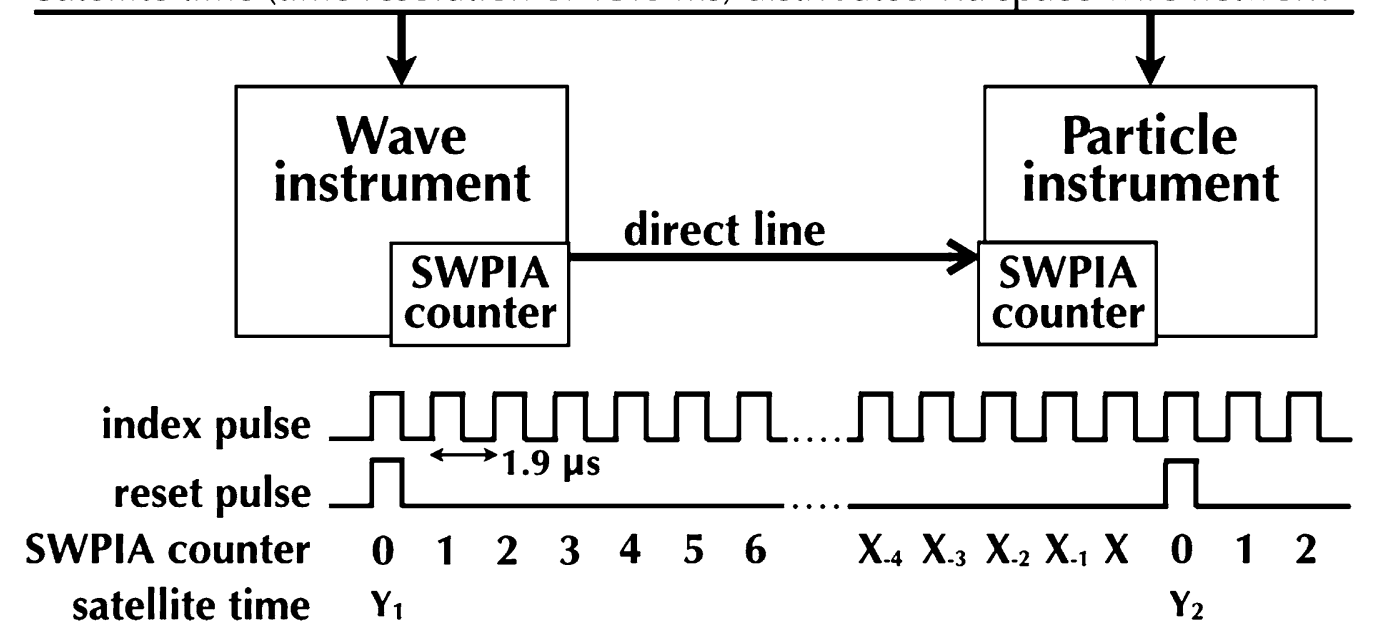

Fig. 4 Schematic diagram of the synchronization of instruments aboard the Arase satellite

of the instruments are not synchronized with each other, a standard clock is generated and distributed by the PWE through the exclusive lines between the PWE and each particle instrument, in order to maintain the accuracy of the relative observation times among the instruments. This clock is called the S-WPIA clock and is configured by dividing the source clock of the PWE. The clock frequency for the S-WPIA clock is $524,288 \mathrm{~Hz}$, which is equivalent to a resolution of $1.907 \mu \mathrm{s}$ (Fig. 4). Since the sampling frequency for waveforms in the PWE is generated from the same source clock as that for the S-WPIA clock, the waveform sampling time is completely synchronized with the time as measured by the S-WPIA clock. Each instrument introduces a counter called the S-WPIA counter, which accumulates counts from the S-WPIA clock. The time index (satellite time) is generated with a time resolution of $15.6 \mathrm{~ms}$ and is distributed by the satellite system. The S-WPIA counter is a 24-bit counter that resets to zero with each distribution of the time index. Thus, the relative observation time is guaranteed by the combination of the time index and the S-WPIA counter with an accuracy of $1.907 \mu \mathrm{s}$ (Fig. 4). Each instrument writes its own observation data attached to the information of the satellite time and the S-WPIA counter.

\section{Operation and output of the S-WPIA}

The S-WPIA computes $W_{\text {int }}$ from the observational data of the PWE, XEP, HEP, MEP-e, and MGF stored on the MDR. Because of the vast amounts of observed raw data for electromagnetic waveforms and individual particle counts, the S-WPIA measurement is intermittent and has a short duration for each orbit of the Arase satellite. First, we set the command to activate the generation of the raw data for each instrument only in the region of interest.
After the observation, by referring to quick-look plots of the PWE, we determine the time interval subject to the computation of the S-WPIA. Then, we set the command for the computation of the stored data observed in the time interval to obtain $W_{\text {int }}, \sigma_{\mathrm{W}}$, and $N$ as functions of $K$, $\alpha$, and $\zeta$. The output of the S-WPIA, $W_{\text {int }}(K, \alpha, \zeta), \sigma_{\mathrm{W}}(K$, $\alpha, \zeta)$, and $N(K, \alpha, \zeta)$, is transferred to the ground, and the raw data used for the S-WPIA output can also be downlinked for verification and investigation of the S-WPIA algorithm. Details of the S-WPIA calculation and the specifications of the S-WPIA software applications are described in Hikishima et al. (submitted to Earth, Planets and Space).

\section{Summary}

In this report, the principle of the WPIA (Fukuhara et al. 2009) and its significance for the direct measurements of wave-particle interactions in the Arase mission were described. The WPIA computes an inner product $W\left(t_{i}\right)=$ $q \boldsymbol{E}\left(t_{i}\right) \cdot \boldsymbol{v}_{i}$, where $t_{i}$ is the detection time for the $i$-th particle, $\boldsymbol{E}\left(t_{i}\right)$ is the wave electric field vector at $t_{i}$, and $q$ and $\boldsymbol{v}_{i}$ represent the charge and the velocity vectors for the $i$-th particle, respectively. Since $W\left(t_{i}\right)$ denotes the gain or the loss of the kinetic energy for the $i$-th particle, summing $W$ for detected particles allows the net amount of the energy exchange in the region of interest to be acquired. By referring to the specifications of the MEP-e and by assuming a count rate of $5000 \mathrm{cps}$ for each detector of the MEP-e, we estimated that the number of particles required to obtain statistically significant results by the S-WPIA can be collected during 18 spin periods.

The implementation of the S-WPIA on the Arase satellite is next described. The S-WPIA was installed on the Arase satellite as a software function running on the 
mission data processor. It uses an electromagnetic field waveform measured by the WFC of the PWE and velocity vectors detected by the MEP-e, HEP, and XEP. The primary goal of the S-WPIA is measuring the energy exchange between the whistler-mode chorus emissions and energetic electrons in the inner magnetosphere. It is essential for the S-WPIA to synchronize instruments with a relative time accuracy that is better than the time period of the plasma wave oscillations. Since the typical frequency of chorus emissions is a few $\mathrm{kHz}$ in the inner magnetosphere, a relative time accuracy better than $10 \mu \mathrm{s}$ should be maintained in order to measure the relative phase angle between wave electromagnetic field and velocity vectors with an accuracy sufficient to correctly detect the sign of $W$. In the Arase satellite, a dedicated system has been developed in order to obtain the required time resolution for inter-instrument communication. Both the time index distributed to all instruments through the satellite system with a time resolution of $15.6 \mathrm{~ms}$ and the S-WPIA clock signal, which is distributed from the PWE every $1.9 \mu$ s to particle instruments through a direct line, are used. The S-WPIA has been successfully implemented on the Arase satellite with instrument specifications and mission networks suitable for the direct measurement of interactions between chorus and energetic electrons in the inner magnetosphere. The S-WPIA software on board the Arase satellite is described in detail in an accompanying paper by Hikishima et al. (submitted to Earth, Planets and Space).

\section{Authors' contributions}

YK and MK contributed theoretical consideration and data analysis. $H K, M H, T T$, and KA contributed discussion of the implementation. YM, YK, SK, TM, NH, AM, $M O, A Y, S Y, S M$, and IS contributed discussion of specifications of instruments.

\section{Author details \\ ${ }^{1}$ Department of Geophysics, Graduate School of Science, Tohoku University, 6-3 Aramaki-aza-aoba, Aoba, Sendai, Miyagi 980-8578, Japan. ${ }^{2}$ Research Institute for Sustainable Humanosphere, Kyoto University, Gokasho, Uji, Kyoto 611-0011, Japan. ${ }^{3}$ ISAS/JAXA, Sagamihara, Kanagawa 229-8510, Japan. ${ }^{4}$ Institute for Space-Earth Environmental Research, Nagoya University, Nagoya, Aichi 464-8601, Japan. ${ }^{5}$ Graduate School of Natural Science and Technology, Kanazawa University, Kakuma, Kanazawa 920-1192, Japan. ${ }^{6}$ Graduate School of Science, The University of Tokyo, Bunkyo-ku, Tokyo 113-0033, Japan. ${ }^{7}$ RDD, JAXA, Tsukuba, Ibaraki 305-8505, Japan. ${ }^{8}$ Osaka University, Toyonaka 560-0043, Japan.}

\section{Acknowledgements \\ The authors express their sincere gratitude for numerous efforts made by all members of the ERG project. This study is supported by Grants-in-Aid for Scientific Research (23224011, JP15H05747, JP15H05815, JP15H03730, JP16H06286, and 17K18798) of Japan Society for the Promotion of Science. This work was also supported by Toray Science and Technology Grant of Toray Science Foundation. This work was carried out by the joint research program of the Institute for Space-Earth Environmental Research (ISEE), Nagoya University. The authors wish to express their sincere appreciations to Emeritus Professor Takayuki Ono for valuable discussion and continuous encourage- ment on this study.}

\section{Competing interests}

The authors declare that they have no competing interests.

\section{Availability of data and materials}

The data used in this paper can be obtained upon request to the corresponding author.

\section{Publisher's Note}

Springer Nature remains neutral with regard to jurisdictional claims in published maps and institutional affiliations.

Received: 31 August 2017 Accepted: 25 December 2017

Published online: 08 January 2018

\section{References}

Buckley AM, Gough MP, Alleyne H, Yearby K, Willis I (2000) Measurement of wave-particle interactions in the magnetosphere using the DWP particle correlator. In: Proceedings of cluster-II workshop, pp 303-306

Ergun RE, Carlson CW, McFadden JP, Clemmons JH, Boehm MH (1991) Langmuir wave growth and electron bunching: results from a wave-particle correlator. J Geophys Res 96:225-238

Ergun RE, McFadden JP, Carlson CW (1998) Wave-particle correlator instrument design. Meas Tech Space Plasmas Part AGU Geophys Monogr 102:325-331

European cooperation for space standardization (ECSS-E-ST-50-12C) (2008) Space engineering, SpaceWire-Links, nodes, routers and networks, European Space Agency

European cooperation for space standardization (ECSS-E-ST-50-51C) (2010) Space engineering, SpaceWire protocol integration, European Space Agency

European cooperation for space standardization (ECSS-E-ST-50-52C) (2010) Space engineering, SpaceWire-Remote memory access protocol, European Space Agency

Fukuhara H, Kojima H, Ueda Y, Omura Y, Katoh Y, Yamanaka H (2009) A new instrument for the study of wave-particle interactions in space: one-chip Wave-Particle Interaction Analyzer. Earth Planets Space 61:765-778. https://doi.org/10.1186/BF03353183

Gough MP, Hardy DA, Oberhardt MR, Burke WJ, Gentile LC, McNeil B, Bounar K, Thompson DC, Raitt WJ (1995) Correlator measurements of megahertz wave-particle interactions during electron beam operations on STS. $J$ Geophys Res 100:21561-21575

Hikishima M, Katoh Y, Kojima H (2014) Evaluation of waveform data processing in Wave-Particle Interaction Analyzer. Earth Planets Space 66:63. https:// doi.org/10.1186/1880-5981-66-63

Kasahara Y, Kasaba Y, Kojima H, Yagitani S, Ishisaka K, Kumamoto A, Tsuchiya F, Ozaki M, Matsuda S, Imachi T, Miyoshi Y, Hikishima M, Katoh Y, Ota M, Shoji M, Matsuoka A, Shinohara I (2018a) The plasma wave experiment (PWE) on board the Arase (ERG) Satellite. Earth Planets Space. https://doi. org/10.1186/s40623-017-0759-3.

Kasahara S, Yokota S, Mitani T, Aasamura K, Hirahara M, Shibano Y, Takashima T (2018b) Medium-Energy Particle experiments - electron analyser (MEP-e) for the Exploration of energization and Radiation in Geospace (ERG) mission. Earth Planets Space. https://doi.org/10.1186/s40623-017-0752-x

Katoh Y (2014) A simulation study of the propagation of whistler-mode chorus in the Earth's inner magnetosphere. Earth Planets Space 66:6. https://doi. org/10.1186/1880-5981-66-6

Katoh Y, Omura Y (2004) Acceleration of relativistic electrons due to resonant scattering by whistler mode waves generated by temperature anisotropy in the inner magnetosphere. J Geophys Res 109:A12214. https://doi. org/10.1029/2004JA010654

Katoh Y, Omura Y (2007) Computer simulation of chorus wave generation in the Earth's inner magnetosphere. Geophys Res Lett 34:L03102. https:// doi.org/10.1029/2006GL028594

Katoh Y, Omura Y (2011) Amplitude dependence of frequency sweep rates of whistler mode chorus emissions. J Geophys Res 116:A07201. https://doi. org/10.1029/2011JA016496

Katoh Y, Omura Y (2013) Effect of the background magnetic field inhomogeneity on generation processes of whistler-mode chorus and broadband hiss-like emissions. J Geophys Res Space Phys 118:4189-4198. https://doi. org/10.1002/jgra.50395 
Katoh Y, Omura Y (2016) Electron hybrid code simulation of whistler-mode chorus generation with real parameters in the Earth's inner magnetosphere. Earth Planets Space 68:192. https://doi.org/10.1186/ s40623-016-0568-0

Katoh Y, Kitahara M, Kojima H, Omura Y, Kasahara S, Hirahara M, Miyoshi Y, Seki K, Asamura K, Takashima T, Ono T (2013) Significance of Wave-Particle Interaction Analyzer for direct measurements of nonlinear wave-particle interactions. Ann Geophys 31:503-512. https://doi.org/10.5194/ angeo-31-503-2013

Kitahara M, Katoh Y (2016) Method for direct detection of pitch angle scattering of energetic electrons caused by whistler mode chorus emissions. J Geophys Res Space Phys. https://doi.org/10.1002/2015JA021902

Kletzing CA, LaBelle J, Bounds SR, Dolan J, Kaeppler SR, Dombrowski M (2017) Phase sorting wave-particle correlator. J Geophys Res Space Phys 122:2069-2078. https://doi.org/10.1002/2016JA023334

Miyoshi Y, Morioka A, Obara T, Misawa H, Nagai T, Kasahara Y (2003) Rebuilding process of the outer radiation belt during the 3 November 1993 magnetic storm: NOAA and EXOS-D observations. J Geophys Res 108(A1):1004. https://doi.org/10.1029/2001JA007542

Omura Y, Furuya N, Summers D (2007) Relativistic turning acceleration of resonant electrons by coherent whistler mode waves in a dipole magnetic field. J Geophys Res 112:A06236. https://doi.org/10.1029/2006JA012243
Omura Y, Katoh Y, Summers D (2008) Theory and simulation of the generation of whistler-mode chorus. J Geophys Res 113:A04223. https://doi. org/10.1029/2007JA012622

Omura Y, Hikishima M, Katoh Y, Summers D, Yagitani S (2009) Nonlinear mechanisms of lower-band and upper-band VLF chorus emissions in the magnetosphere. J Geophys Res. https://doi.org/10.1029/2009JA014206

Shoji M, Miyoshi Y, Katoh Y, Keika K, Angelopoulos V, Kasahara S, Asamura K, Nakamura S, Omura Y (2017) Ion hole formation and nonlinear generation of electromagnetic ion cyclotron waves: THEMIS observations. Geophys Res Lett. https://doi.org/10.1002/2017GL074254

Summers D, Omura Y (2007) Ultra-relativistic acceleration of electrons in planetary magnetospheres. Geophys Res Lett 34:L24205. https://doi. org/10.1029/2007GL032226

Summers D, Thorne RM, Xiao F (1998) Relativistic theory of wave-particle resonant diffusion with application to electron acceleration in the magnetosphere. J Geophys Res 103:20487

\section{Submit your manuscript to a SpringerOpen ${ }^{\circ}$ journal and benefit from:}

- Convenient online submission

- Rigorous peer review

- Open access: articles freely available online

- High visibility within the field

- Retaining the copyright to your article

Submit your next manuscript at $\boldsymbol{\nabla}$ springeropen.com 